\title{
Review
}

\section{Gestational diabetes - Diagnostic and therapeutic novelties}

\author{
Marinela Chioveanu ${ }^{1}$, Oana-Denisa Bălălău ${ }^{2,3}$, Romina-Marina Sima ${ }^{2,3}$, Liana Pleș ${ }^{2,3}$, \\ Cristian Bălălău², Anca-Daniela Stănescu ${ }^{2,3}$
}

${ }^{1}$ Nicolae Paulescu National Institute of Diabetes, Bucharest, Romania

${ }^{2}$ Carol Davila University of Medicine and Pharmacy, Bucharest, Romania

${ }^{3}$ St. Ioan Clinical Emergency Hospital - Bucur Maternity, Bucharest, Romania

\begin{abstract}
The prevalence of diabetes during pregnancy has been steadily increasing in the US, mainly gestational diabetes (approximately $90 \%$ of the cases). The other types of diabetes during pregnancy are type 1 and type 2 diabetes mellitus. Pre-existing type 2 diabetes mellitus represents approximately $8 \%$ of all types of diabetes during pregnancy. The increase in the prevalence of gestational diabetes occurred in parallel with the increase in the prevalence of obesity.

The diagnosis of gestational diabetes is made either through one-step or two-step strategy in pregnant women between 24 and 28 weeks of gestation. Diabetes screening is also recommended at the first prenatal visit to women with increased risk factors for diabetes. The management of diabetes during pregnancy involves nutritional medical therapy and/ or pharmacological treatment, in order to achieve the glycemic targets that have been associated with the lowest risk of maternal and fetal complications.

Considering the possibility of diabetic complications (retinopathy) during pregnancy, the screening of women with pre-existing diabetes is also necessary. Moreover, gestational diabetes has been associated with an increase in the risk of developing maternal type 2 diabetes, in approximately $50-70 \%$ of the cases, after the age of $15-25$ years. Therefore, it is recommended to test women with gestational diabetes, between 4-12 weeks' post-partum, using the oral glucose tolerance test (OGTT). If the results are normal, the test should be performed every 1 to 3 years.
\end{abstract}

Keywords $\quad:$ gestational diabetes, diabetes complications, glycemic targets

Highlights $\quad \checkmark$ The active screening of women with risk factors for diabetes, the early diagnosis and the appropriate treatment are required to achieve optimal glycemic control in pregnancy.

$\checkmark$ A proper treatment of gestational diabetes leads to a decrease in the rate of perinatal mortality and hospitalization in intensive care units, improving fetal and maternal outcomes.

To cite this article: Chioveanu M, Bălălău OD, Sima RM, Pleș L, Bălălău C, Stănescu AD. Gestational diabetes - Diagnostic and therapeutic novelties. J Clin Invest Surg. 2019; 4(2): 66-71. DOI: $10.25083 / 2559.5555 / 4.2 / 66.71$ 


\section{Introduction}

Gestational diabetes is the diabetes diagnosed during pregnancy, in the $2 \mathrm{nd}$ or 3rd trimester, which is neither type 1 diabetes nor pre-existing type 2 diabetes. The diabetes diagnosed in the first trimester of pregnancy is defined as pre-existing diabetes mellitus (1-4).

The association between pregnancy and diabetes can occur in two situations: if the pregnancy occurs in women with known type 1 or type 2 diabetes, this is called pregestational diabetes; on the other hand, the diabetes diagnosed in the $2 \mathrm{nd}$ or $3 \mathrm{rd}$ trimester of pregnancy is called gestational diabetes $(1,4)$.

The prevalence of pre-existing diabetes has increased in the past decade, especially as a result of the increased prevalence of type 2 diabetes mellitus. For these women, the main cause of morbidity and mortality in children is represented by major congenital malformations (1).

Therefore, these women should be informed and educated about these risks, about the need for optimal glycemic control before the preconception phase, and the contraceptive and family planning options. Preconception counselling improved maternal and fetal outcomes in women with pre-existing diabetes $(5,6)$.

Gestational diabetes represents about $90 \%$ of all the pregnancies associated with diabetes mellitus. The increase in the prevalence of gestational diabetes occurred in parallel with the prevalence of obesity. The prevalence of hyperglycemia during pregnancy has been estimated at $16.9 \%$ (7).

Pre-existing diabetes screening is recommended at the first prenatal visit in women with increased risk factors for diabetes. Screening for gestational diabetes occurs between 24 and 28 weeks of pregnancy. Then, if they have been diagnosed with gestational diabetes, another test is recommended between 4-12 weeks postpartum (1-4).

The first evidence that screening, diagnosis and treatment of hyperglycemia in women not previously known with diabetes improved the outcomes was emphasized by O'Sullivan et al. in the 1960s. They proposed several diagnostic criteria for gestational diabetes based on a 3-h 100g oral glucose tolerance test (OGTT). They also tested the effectiveness of antidiabetic treatment (diet or insulin) in pregnant women. They demonstrated that the fetal macrosomia rate was $4.3 \%$ in the intervention group compared to $13.1 \%$ in the control group (3).

\section{Discussions}

It has been demonstrated that women with preexisting diabetes mellitus and their children have a double risk of serious injury at birth, a triple risk of Cesarean delivery, and a quadruple risk of admission to the intensive care units.

Studies on women with preexisting diabetes have revealed higher rates of complications compared to the general population, including perinatal mortality, congenital malformations, hypertension, preterm delivery, large-for-gestational age (LGA) infants, Cesarean delivery and other neonatal morbidities $(6,8)$.

Moreover, the incidence of pediatric diabetes was higher in children born by mothers with gestational diabetes than by mothers without gestational diabetes, according to a study published in April 2019 (9-11).

A child with macrosomia or a large child for the gestational age is at increased risk for the onset of shortterm complications, but it can also increase the risk of longterm complications such as childhood obesity, diabetes, or high blood pressure. Fetal macrosomia occurs in 15-45 \% of diabetic pregnancies. Also, large-for-gestational-age infants should be screened for hypoglycemia (5).

In order to minimize the occurrence of these complications, it is important that these women have a good glycemic control prior to pregnancy. According to the American Diabetes Association 2019, a good glycemic control is defined by a glycosylated hemoglobin $<6.5 \%$, the value below which the risk of congenital anomalies, preeclampsia, macrosomia, and other complications decreases. Also, continuous glycemic monitoring (CGM) has improved glycemic control and neonatal outcomes in these women $(1,4)$.

Optimal glycemic control prior to pregnancy has been associated with a $70 \%$ decrease in maternal and fetal complications. Some observational studies have revealed an increased risk of anencephaly, microcephaly, congenital heart disease, caudal regression, directly proportional to increased glycosylated hemoglobin during the first 10 weeks of pregnancy (1).

Moreover, women with pre-existing diabetes should be informed about the risk of developing/ worsening diabetic retinopathy and other diabetic complications. Diabetic retinopathy is the main cause of vision loss in women aged between 24 and 64 years. Because of this risk, the ophthalmic control is recommended before pregnancy (in the first trimester, every trimester, and 1 year postpartum).

At the first prenatal visit of women with preexisting diabetes, it is recommended to re-evaluate potentially teratogenic drugs, to assess comorbidities such as hypertension, renal disease, the occurrence of diabetic neuropathy and the presence of cardiovascular disease. The rate of preeclampsia has been found to correlate with the level of glycemic control. 
Albuminuria and overt nephropathy are associated with increased risk of maternal and fetal complications. Given the risk of deterioration of the renal function, an estimated glomerular filtration rate (eGFR) should be used before pregnancy to determine the risk of adverse outcomes. Additional folic acid is also recommended, given that obesity, which is more commonly associated with type 2 diabetes, has been associated with lower levels of folic acid than in the general population (1-4).

The diagnostic criteria for type 1 diabetes mellitus and type 2 diabetes mellitus are as follows:

1. Fasting glycaemia $>126 \mathrm{mg} / \mathrm{dL}$, or

2. 2-h OGTT glycaemia $>200 \mathrm{mg} / \mathrm{dL}$, or

3. Glycated hemoglobin $>6.5 \%$, or

4. Symptomatic patient and random glycaemia > 200 $\mathrm{mg} / \mathrm{dL}$.

Between $3 \%$ and $20 \%$ of the pregnant women develop gestational diabetes, the percentage varying according to the occurrence of risk factors. The HAPO study revealed the association between plasma glucose and adverse pregnancy outcomes, this association being independent of other risk factors $(1,9)$.

Pregnant women diagnosed with pre-diabetes before pregnancy are at increased risk of developing gestational diabetes, and should benefit from screening in the first trimester of pregnancy.

The risk factors for the development of gestational diabetes are as follows:

1. Mother's age over 35 years

2. High-risk Ethnicity (African, Arab, Asian, Hispanic, Indigenous, or South Asian)

3. Under corticosteroid medication

4. Obesity (and body mass index higher than or equal to $30 \mathrm{~kg} / \mathrm{m} 2)$

5. Pre-diabetes

6. Gestational diabetes during a previous pregnancy

7. Birth given to a baby whose weight was higher than 4 $\mathrm{kg}$

8. A parent, a brother or a sister with type 2 diabetes

9. Polycystic ovary syndrome or acanthosis nigricans

Some risk factor associated with the lowest incidence of gestational diabetes are:

1. Age $<25$ years

2. Normal weight before pregnancy

3. Member of an ethnic group with a low prevalence of diabetes

4. No known diabetes in first-degree relatives

5. No history of abnormal glucose tolerance

6. No history of poor obstetric outcome $(2,3)$.
Diagnosing gestational diabetes is essential, given the increased risk in the mother and the infant associated with gestational diabetes and, on the other hand, the benefits obtained by the optimal glycemic control. The screening of gestational diabetes will be performed in the 24th-28th week of pregnancy. The best method of screening for gestational diabetes is controversial.

The method of screening can be either a sequential 2step or a 1-step process:

The one-step strategy includes the test with 75 grams of oral glucose after 8 hours of fasting. This is the test performed between 24 to 28 weeks of gestation and it results positive if one of the following criteria is met:

1. Fasting glycaemia greater than or equal to $92 \mathrm{mg} / \mathrm{dl}$

2. 1-hour glycaemia greater than or equal to $180 \mathrm{mg} / \mathrm{dl}$

3. 2-hour glycaemia greater than or equal to $153 \mathrm{mg} / \mathrm{dl}$

The two-step strategy includes the administration of 50 g oral (non-fasting) glucose with glucose measurement 1 hour after administration. If blood glucose at 1 hour is greater than or equal to $130 \mathrm{mg} / \mathrm{dl}, 135 \mathrm{mg} / \mathrm{dl}$, or $140 \mathrm{mg} /$ $\mathrm{dl}, 100 \mathrm{~g}$ of glucose will be administered after 8 hours of fasting. The diagnosis of gestational diabetes is made if 2 of the next 4 criteria are met:

1. Fasting glycaemia greater than or equal to $95 \mathrm{mg} / \mathrm{dl}$

2. Blood glucose at 1 hour greater than or equal to 180 $\mathrm{mg} / \mathrm{dl}$

3. Blood glucose at 2 hours greater than or equal to 155 $\mathrm{mg} / \mathrm{dl}$

4. Glucose at 3 hours greater than or equal to $140 \mathrm{mg} / \mathrm{dl}$

Other tests (glycated hemoglobin, random postprandial or fasting blood sugar levels) are not recommended because of their low sensitivity $(1,8)$.

Fasting and postprandial self-monitoring of blood glucose are recommended in both gestational diabetes mellitus and pre-existing diabetes during pregnancy to achieve glycemic control. Some women with preexisting diabetes should also test blood glucose pre-prandially, in order to adjust their pre-fast acting insulin dosage.

Glycemic targets are the same for both gestational diabetes and pre-existing type 1 or type 2 diabetes mellitus. These are the following:

1. Fasting/ pre-prandial glucose concentrations, $95 \mathrm{mg} /$ dL

2. 1-hour postprandial, $140 \mathrm{mg} / \mathrm{dL}$

3. 2-hour postprandial, $120 \mathrm{mg} / \mathrm{dL}$

After the diagnosis, treatment starts with medical nutrition therapy, physical activity, and weight management depending on the pre-gestational weight (1). 
Most women can achieve euglycemia through nutritional therapy alone, but $30 \%$ will require drug therapy $(12,13)$. Moreover, it has been demonstrated that insulin treatment administered in case the target blood glucose concentrations are not met through diet resulted in the reduction of preeclampsia, shoulder dystocia and birth weight $>4000 \mathrm{~g}$. Weight loss before pregnancy has been shown to be the most effective intervention that improved medical comorbidities, such as diabetes and hypertension.

Some studies suggest that $70-85 \%$ of the women diagnosed with gestational diabetes can control it with lifestyle modification alone. On the other hand, weight gain more than recommended has a higher risk of preeclampsia, delivery through Cesarean section, macrosomia and largefor-gestational-age fetus (14).

Excessive gestational weight gain is characterized by higher birth weight infants. Because pre-pregnancy BMI and glycemic control have independent effects on fetal growth, the education and management of this women are very important. According to a recent review, $47 \%$ of the pregnant women in the United States gain more weight than recommended.

Pre-pregnancy body mass index seems to be a more important risk factor for adverse maternal-fetal outcomes than weight gain during pregnancy, according to a metaanalysis (13).

In a review from 2017, it was demonstrated that gestational weight gain below recommendations was associated with a higher risk of small-for-gestational-age and preterm birth and lower risk of large-for-gestationalage and macrosomia, while gestational weight gain above recommendations was associated with higher risks of large for gestational age, macrosomia and Cesarean delivery (12).

In a study published in May 2019, it was demonstrated that the risk of obesity in children increases at glycemic levels during pregnancy below those used to diagnose gestational diabetes, and also that the effect of gestational diabetes on obesity in children is higher than in women with obesity (10).

In preexisting diabetes mellitus, the previous diet will be maintained during pregnancy, with the permanent caloric adaptation, depending on the initial weight before pregnancy, on the weight evolution and on the age of the pregnancy. In overweight/ obese pregnancies, a moderate low caloric intake is recommended, but no weight loss is indicated $(1,4)$.

With regard to women with gestational diabetes, there is no consensus on optimal caloric intake. Nutrition therapy should be designed to promote adequate nutritional intake without ketosis, achievement of glycemic goals, appropriate fetal growth and maternal weight gain. The guidelines recommend a minimum carbohydrate intake of $175 \mathrm{~g} /$ day, a minimum of 71 grams of protein/ day and 28 grams of fiber/ day, with the reduction of refined sugars, the elimination of alcohol and adequate fluid intake.

Physical activity of moderate intensity should be maintained and carried out constantly, but adapted to the evolution of the pregnancy. In combination with nutritional intervention, physical activity appears to be more effective for gestational diabetes management than gestational diabetes prevention (1-4).

\section{Pharmacological Therapy}

Insulin is the first-line agent used in the treatment of gestational diabetes, along with nutritional intervention and physical activity. The choice of insulin regimen is based on the patient's individual glucose profile. Other oral antidiabetic agents, such as sulphonylureas, cross the placenta and are associated with neonatal hypoglycemia, and metformin increased the risk of prematurity, even if it is associated with a lower risk of neonatal hypoglycemia.

For pregnant women diagnosed with pre-existent diabetes, an intensive insulin therapy regimen (basal bolus regimen) or insulin pump therapy may be used. In these cases, intensive glycemic monitoring (approximately 6-10 times/ day) is required to adjust insulin doses to ensure an optimal glycemic control and to avoid hypoglycemia. The insulin dose depends on the age of the pregnancy and the weight of the mother.

Several studies have indicated a possible benefit of adding metformin to insulin therapy in women with preexisting type 2 diabetes, but there are still no clear benefits of prescribing metformin in these people.

In pregnant women with gestational diabetes, insulin therapy will be initiated when the diet and physical activity fail to ensure glycemic targets. Different insulin therapy regimens may be used, but the presence of hyperglycemia, both pre-prandial and post-prandial, requires the administration of a combined (basal-bolus) regimen. Frequent glycemic self-monitoring is also recommended in order to achieve the optimal glycemic target.

Due to the complexity of insulin management in pregnancy, referral to a specialized center providing multidisciplinary care, including a maternal-fetal medicine specialist, an endocrinologist, or another practitioner experienced in the management of pregnancy in women with preexisting diabetes, a dietitian, a nurse, is recommended if this is available. 
In most situations, gestational diabetes will resolve after birth, but there are situations where there may be various degrees of glucose intolerance. It is recommended to test women between 4-12 weeks' post-partum with an OGTT, with subsequent testing at 3 years (1-4).

According to a study published in May 2019, the infants of mothers who have insulin- treated diabetes mellitus, are at a higher risk for preterm and macrosomia than the infants of mothers with gestational diabetes (15, 16).

\section{Conclusions}

Considering the increased risk of morbidity and mortality associated with diabetes in pregnancy, the active screening of women with risk factors, the early diagnosis of diabetes, and the appropriate treatment are required to achieve optimal glycemic control.

The treatment of gestational diabetes resulted in a significant decrease in the risk of macrosomia, large for gestational age and shoulder dystocia. Moreover, the rate of perinatal mortality and hospitalization in intensive care units were lower in treated women.

Last but not least, monitoring/ self-monitoring of glycaemia is the key to gestational diabetes therapy, improving fetal and maternal outcomes.

\section{Conflict of interest disclosure}

There are no known conflicts of interest in the publication of this article. The manuscript was read and approved by all authors.

\section{Compliance with ethical standards}

Any aspect of the work covered in this manuscript has been conducted with the ethical approval of all relevant bodies and that such approvals are acknowledged within the manuscript.

\section{References}

1. Burlina $\mathrm{S}$, Dalfrà $\mathrm{MG}$, Visentin $\mathrm{S}$, Valentini $\mathrm{R}$, Capovilla F, Lapolla A; Training Experience Group. Team management of gestational diabetes: a training experience. Acta Diabetol. 2017; 54(9): 881-883. DOI: 10.1007/s00592-017-1003-0.

2. Halimeh R, Melchiorre K, Thilaganathan B. Preventing term stillbirth: benefits and limitations of using fetal growth reference charts. Curr Opin Obstet Gynecol. 2019; 31(6): 365-374.

3. Vestgaard M, Christensen AS, Viggers L, Lauszus FF. Birth weight and its relation with medical nutrition therapy in gestational diabetes. Arch Gynecol Obstet. 2017; 296(1): 35-41. DOI: 10.1007/s00404-017-43967.

4. Mack LR, Tomich PG. Gestational Diabetes: Diagnosis, Classification, and Clinical Care. Obstet Gynecol Clin North Am. 2017; 44(2): 207-217. DOI: 10.1016/j.ogc.2017.02.002.

5. Lawrence RL, Wall CR, Bloomfield FH. Prevalence of gestational diabetes according to commonly used data sources: an observational study. BMC Pregnancy Childbirth. 2019; 19(1): 349. DOI: 10.1186/s12884019-2521-2.

6. Badon SE, Enquobahrie DA, Wartko PD, Miller RS, Qiu C, Gelaye B, Sorensen TK, Williams MA. Healthy Lifestyle During Early Pregnancy and Risk of Gestational Diabetes Mellitus. Am J Epidemiol. 2017; 186(3): 326-333. DOI: 10.1093/aje/kwx095.

7. Petrović O, Belci D. A critical appraisal and potentially new conceptual approach to screening and diagnosis of gestational diabetes. J Obstet Gynaecol. 2017; 37(6): 691-699. DOI: 10.1080/01443615.2017.1306692.

8. Minooee S, Ramezani Tehrani F, Rahmati M, Mansournia MA, Azizi F. Diabetes incidence and influencing factors in women with and without gestational diabetes mellitus: A 15year populationbased follow-up cohort study. Diabetes Res Clin Pract. 2017; 128: 24-31. DOI: 10.1016/j.diabres.2017.04.003.

9. Zheng $\mathrm{J}$, Wang $\mathrm{H}$, Ren $\mathrm{M}$. Influence of exercise intervention on gestational diabetes mellitus: a systematic review and meta-analysis. $J$ Endocrinol Invest. 2017; 40(10): 1027-1033. DOI: 10.1007/s40618-017-0673-3

10. Battarbee AN, Venkatesh KK, Aliaga S, Boggess KA. The association of pregestational and gestational diabetes with severe neonatal morbidity and mortality. J Perinatol. 2019 Oct 7. DOI: 10.1038/s41372-0190516-5.

11. Kosinska-Kaczynska K, Szymusik I, Kaczynski B, Wielgos M. Observational study of associations between gestational weight gain and perinatal outcomes in dichorionic twin pregnancies. Int $J$ Gynaecol Obstet. 2017; 138(1): 94-99. DOI: 10.1002/ijgo.12171.

12. Yang GR, Dye TD, Li D. Effects of pre-gestational diabetes mellitus and gestational diabetes mellitus on macrosomia and birth defects in Upstate New York. Diabetes Res Clin Pract. 2019; 155: 107811. DOI: 10.1016/j.diabres.2019.107811.

13. Taschereau-Charron A, Da Silva MS, Bilodeau JF, Morisset AS, Julien P, Rudkowska I. Alterations of 
fatty acid profiles in gestational diabetes and influence of the diet. Maturitas. 2017; 99: 98-104. DOI: 10.1016/j.maturitas.2017.01.014.

14. Rogozińska E, Zamora J, Marlin N, Betrán AP, Astrup A, Bogaerts A, Cecatti JG, Dodd JM, Facchinetti F, Geiker NRW, Haakstad LAH, Hauner H, Jensen DM, Kinnunen TI, Mol BWJ, Owens J, Phelan S, Renault KM, Salvesen KA, Shub A, Surita FG, Stafne SN, Teede H, van Poppel MNM, Vinter CA, Khan KS, Thangaratinam S; International Weight Management in Pregnancy (i-WIP) Collaborative Group. Gestational weight gain outside the Institute of Medicine recommendations and adverse pregnancy outcomes: analysis using individual participant data from randomised trials. BMC Pregnancy Childbirth. 2019; 19(1): 322. doi: 10.1186/s12884-019-2472-7.

15. Schoenaker DAJM, Mishra GD. Association Between Age at Menarche and Gestational Diabetes Mellitus: The Australian Longitudinal Study on Women's Health. Am J Epidemiol. 2017; 185(7): 554-561. DOI: 10.1093/aje/kww201.

16. Shiqiao H, Bei X, Yini Z, Lei J. Risk factors of gestational diabetes mellitus during assisted reproductive technology procedures. Gynecol Endocrinol. 2019 Aug 21: 1-4. DOI: 10.1080/09513590.2019.1648418. 